\title{
Experimental investigation on parametric excitation of plasma oscillations in Josephson tunnel junctions
}

Bak, Christen Kjeldahl; Kofoed, Bent; Pedersen, Niels Falsig; Særmark, Knud

Published in:

I E E E Transactions on Magnetics

Publication date:

1975

Document Version

Publisher's PDF, also known as Version of record

Link back to DTU Orbit

Citation (APA):

Bak, C. K., Kofoed, B., Pedersen, N. F., \& Særmark, K. (1975). Experimental investigation on parametric excitation of plasma oscillations in Josephson tunnel junctions. I E E E Transactions on Magnetics, 11(2), 829833.

\section{General rights}

Copyright and moral rights for the publications made accessible in the public portal are retained by the authors and/or other copyright owners and it is a condition of accessing publications that users recognise and abide by the legal requirements associated with these rights.

- Users may download and print one copy of any publication from the public portal for the purpose of private study or research.

- You may not further distribute the material or use it for any profit-making activity or commercial gain

- You may freely distribute the URL identifying the publication in the public portal 


\title{
EXPERIMENTAL INVESTIGATION ON PARAMETRIC EXCITATION
} OF PLASMA OSCILLATIONS IN JOSEPHSON TUNNEL JUNCTIONS

\author{
C. K. Bak, B. Kofoed, N. F. Pedersen, and K. Saermark*
}

\section{ABSTRACT}

Experimental evidence for subharmonic, parametric excitation of plasma oscillations in Josephson tunnel junctions is presented. The experiments described are performed by measuring the microwave power necessary to switch a Josephson tunnel junction biased in the zero voltage state to a finite voltage state.

\section{INTRODUCTION}

The use of a Josephson junction as a parametric amplifier has been investigated previously, theoretically $1,2,3$ as well as experimentally. $2,4,5$. In the Refs. $1,2,4,5$ the Josephson frequency $\omega_{0}=2 \mathrm{eV}_{0} / \hbar$ is used as the pump frequency and an external cavity is used as the input circuit. Here e is the electron charge, $2 \pi \hbar$ is Planck's constant, and $V_{o}$ is the d. c. voltage across the junction. In Ref. 3 the possibility of using the plasma frequency in a Josephson junction as the input circuit and an external signal as the pump has been investigated theoretically, and it was suggested that parametric excitation of the plasma oscillations should be observable in a Josephson thin film junction, but less likely in point contacts. It is well known, that a Josephson junction operating in the zero voltage mode may be assigned an inductance given by

$$
L=\frac{\hbar}{2 \mathrm{e}}\left(\mathrm{I}_{\mathrm{dc}}^{\max } \cos \phi_{\mathrm{o}}\right)^{-1}, \sin \phi_{\mathrm{o}}=\mathrm{I}_{\mathrm{dc}} / \mathrm{I}_{\mathrm{dc}}^{\max } \text {. }
$$

The plasma resonance $\omega_{\mathrm{p}}$ is formed with the junction
capacitance $C$

$$
\omega_{p}^{2}=\frac{2 e}{\hbar C} I_{d c}^{\max } \cos \phi_{O}=\left(\omega_{p}, \max \right)^{2} \cos \phi_{0} .
$$

Using the resistive shunt model and neglecting the $\cos \phi$-term we may calculate the Q-factor

$$
\begin{aligned}
& \mathrm{Q}=\mathrm{R} / \omega_{\mathrm{p}} \mathrm{L}=\mathrm{RI} \mathrm{I}_{\mathrm{dc}}^{\max } \frac{2 \mathrm{e}}{\hbar} \cdot \frac{1}{\omega_{\mathrm{p}}} \cos \phi_{o} \\
& \mathrm{Q}=\left(\omega_{\mathrm{g}} / \omega_{\mathrm{p}}\right) \cos \phi_{\mathrm{o}}, \omega_{\mathrm{g}}=\mathrm{RI} \mathrm{m}_{\mathrm{dc}}^{\max } \frac{2 \mathrm{e}}{\hbar} .
\end{aligned}
$$

From Ref. 3 we know that the threshold for onset of plasma oscillations at $\omega_{p}$ by applying $\omega_{R F}=2 \omega_{p}$ may be

$$
\alpha \simeq \frac{2}{\mathrm{Q}} \frac{1}{\operatorname{tg} \phi_{\mathrm{O}}}, \alpha=\frac{2 \mathrm{e} \mathrm{V}_{\mathrm{RF}}}{\hbar \omega_{\mathrm{RF}}} .
$$
Inserting $Q=\left(\omega_{\text {o }} / \omega_{p}\right) \cos \phi_{o}$ and $2 \omega_{p}=\omega_{R F}$ we obtain the
threshold $R F$ voltage

Manuscript received September 30, 1974.

* Physics Laboratory I, The Technical University of Denmark, DK-2800 Lyngby, Denmark.

$$
V_{R F}=\frac{\hbar}{2 e}\left(4 \omega_{p}^{2} / \omega_{g}\right)\left(\sin \phi_{o}\right)^{-1}
$$

From measurements on a Josephson junction analog we know, that the amplitude of the plasma oscillations is of the same order of magnitude as the threshold value of $V_{R F}$. Thus, we obtain the available power at $\omega_{\mathrm{p}}$

$$
\mathrm{P} \simeq \frac{1}{8} \frac{\mathrm{V}_{\mathrm{RF}}{ }^{2}}{\mathrm{R}}
$$
Using the above expression for $V_{R F}$ and $\frac{2 e}{\hbar} R I_{d c}^{\max }=\omega_{g}$
we get

$$
P=\frac{\hbar}{2 \mathrm{e}}\left(\omega_{\mathrm{p}}^{4} / \omega_{\mathrm{g}}^{3}\right) 2 \mathrm{I}_{\mathrm{dc}}^{\max }\left(\sin \phi_{\mathrm{o}}\right)^{-2}
$$

A typical junction with $I_{d c}^{\max }=5 \mathrm{~mA}$ at $f_{\mathrm{p}}=5 \mathrm{GHz}$ and $\sin \phi_{\mathrm{O}}=0.5$ yields

$$
\mathrm{P} \simeq 0.5 \cdot 10^{-13} \mathrm{~W} \text { corresponding to }-103 \mathrm{dbm} \text {. }
$$

This is just the sensitivity limit for an usual wideband spectrum analyzer like the HP $8551 \mathrm{~B}$, so there is no margin for cable and mismatch losses. It explains why initial attempts to directly observe the excitation failed.

\section{EXPERIMENTAL PROCEDURE}

According to the simple theory $I_{d c}^{\max }$ is proportional to $\mathrm{J}_{\mathrm{o}}(2 \mathrm{eV}$ rf $/ \hbar \omega)$ where $\mathrm{J}_{\mathrm{o}}$ is the Bessel function of order zero. Measurement of $I_{\mathrm{dc}}^{\max }$ as function of the square root of the applied microwave power should give this dependence. 7 In general a remarkable variety of singularities and hysteresis effects have been observed for example as reported by Dahm et al. ${ }^{8}$

In tracing the IV-characteristic on an oscilloscope certain intervals of the dc-Josephson current showed instabilities with applied microwaves. Biasing the junction in these intervals for definite power settings was impossible. It is claimed that two of these instabilities are due to the plasma resonance and a parametric excitation of the plasma resonance respectively. In order to make the measurements in a reproducible way the junction was biased with a fixed dc-current, $I_{d c}$, at zero voltage without any microwaves present. The microwave power was then gradually increased until the junction switched to a voltage different from zero. An example of measurements performed in this way is shown in Fig. 1. Here the $\mathrm{db}$-reading of the calibrated attenuator when the junction switched is plotted as function of the dc-bias current, $I_{d c}$, the microwave frequency being kept constant during the measurements. Since it is the $\mathrm{db}$-reading of the inserted attenuator which is plotted 
a maximum point in the curve in Fig. 1 represents a point where the junction demands minimal microwave power for switching to a voltage state. The values of the dc-Josephson current, $I_{d c}^{R}$, at the maximum points are used in the analysis. In order to compare the measurements to similar measurements performed on a Josephson junction analog the square root of the applied microwave power as function of the dcJosephson current has also been plotted. An example is shown in Fig. 2a. In the following we shall call a curve of this type for a "switching curve". Recently Balkashin et al. 10 have reported on a similar curve measured in a different way by keeping the microwave power constant and increase the dc-current until the junction switched.

Curves like the ones which have been shown in Fig. 1 and in Fig. 2a have been measured for a number of microwave frequencies in the intervals $2-4 \mathrm{GHz}$ and $8.5-11 \mathrm{GHz}$.

Measurements as described have been performed on several thin-film Josephson junctions of the type $\mathrm{Sn}-\mathrm{Sn}_{\mathrm{x}} \mathrm{O}-\mathrm{Sn}$ and $\mathrm{Nb}-\mathrm{Nb}_{\mathrm{x}} \mathrm{O}_{\mathrm{y}}-\mathrm{Pb}$. All the junctions behaved in the same manner in full agreement with the discussion in section III.

\section{DISCUSSION OF EXPERIMENTAL RESULTS}

Central to the discussion is the Josephson plasma frequency (1). In the junction experiments we assume the applied microwaves to be a current source. When the applied microwave frequency is equal to the plasma frequency $\omega \simeq \omega$ the junction $r . f$. voltage is enhanced, and the junction will require comparatively lower microwave power for switching than for $\omega \neq \omega$. Hence, minima in the switching curve may be expected when the applied frequency is equal to the plasma frequency and, possibly, to harmonics and subharmonics of $\omega_{\mathrm{p}}$.

Figure 2 a shows a typical experimental switching curve obtained the way described above. The curve shows the square root of the $r$.f. power as a function of the dc-bias current, $I_{d c}$. The applied frequency is $10.897 \mathrm{GHz}$ and the junction has $I_{\mathrm{dc}}^{\max }=6.11 \mathrm{~mA}$ and $\mathrm{a}$ normal state resistance $R=0.1 \mathrm{ohm}$. Three character istic structures are observed in Fig. $2 \mathrm{a}$, and labelled $\omega / \omega_{p}=2,3 / 2$, and 1 respectively. This labelling will be described in detail below. Figure $2 \mathrm{~b}$ is a similar switching curve obtained for a Josephson junction analog; ${ }^{9}$ again the analog is biased in the zero voltage mode and an r.f. signal is applied until it switches to a voltage state. The analog parameters are given in the caption for Fig. $2 \mathrm{~b}$. A careful investigation of the analog voltage waveform on an oscilloscope shows that the observed structures are related to a large signal plasma resonance--subject to the condition $\omega=\omega_{\mathrm{p}}--$ and a parametric, subharmonic excitation of the plasma resonance ${ }^{3}$ subject to the condition $\omega_{p}=\omega \cdot 1 / 2$. In addition the analog experiments show the usual harmonic resonances--subject to the conditiin $n \omega=\omega_{p}$.

In order to unambigously identify the resonances in the real junctions two methods were employed. One method was to obtain switching curves for a wide frequency range covering both harmonic and subharmonic generation and then determine the indexing that would fit all of the observed structures. An example of this method is shown in Fig. 3, which shows a plot of three frequencies in the $8.5-11 \mathrm{GHz}$ range and two frequencies in the $2-4 \mathrm{GHz}$ range. For each frequency the dc-current, $I_{\mathrm{dc}}^{R}$, corresponding to the pronounced structures (minima in the switching curves) is read and the quantity $\sqrt{\cos \phi_{0}}$ (proportional to the plasma frequency, Eq. (1)) is calculated as

$$
\sqrt{\cos \phi_{0}}=\sqrt[4]{1-\left(\frac{I_{d c}^{R}}{I_{d c}^{\max }}\right)^{2}}
$$

This plot gives a maximum plasma frequency of 12.6 $\mathrm{GHz}$ and the series $\omega / \omega_{\mathrm{p}}=2,3 / 2,1,1 / 2,1 / 3$, and $1 / 4$ can be identified.

The other method was to determine independently the magnitude of the plasma frequency by measuring the capacitance and inserting in Eq. (1). For the $\mathrm{Nb}-\mathrm{Nb} \mathrm{O}_{\mathrm{y}}-\mathrm{Pb}$ junction $\left(\mathrm{R}=0.05 \mathrm{ohm}, \mathrm{I}_{\mathrm{dc}}^{\max }=3.3 \mathrm{~mA}\right.$ ) the capacitance was determined by measuring the capacitance of a high resistance $(R=100 \mathrm{ohm})$ junction from the same batch directly and extrapolating as done for example in Ref. 11. The results of the two methods agreed within a few percent. In any case an improper indexing would give a factor of 4 in the capacitance-far beyond the experimental uncertainty. Figure 4 shows the results for the three samples that were most extensively studied. The ordinate is the frequency normalized to the plasma frequency. The straight lines correspond to $\omega / \omega_{p}=2,3 / 2,1,1 / 2,1 / 3$, and $1 / 4$, and are not fitted to the data points.

We add that in one case the plasma frequency was changed by applying a magnetic field. The structure in the switching curve shifted in qualitative agreement with the magnetic field dependence of the plasma frequency. 12

The excitation of the plasma resonance by harmonics of the applied microwave signal is well understood and have been reported earlier. 8,12 What is new in the present experiments is the excitation of the plasma resonance by means of subharmonic generation, which has been theoretically predicted earlier. ${ }^{3}$. The proposed mechanism is a parametric excitation of the plasma resonance when the applied microwave have a frequency $\omega \simeq 2 \omega_{p}$. For $\omega \lesssim 2 \omega_{p}$ this parametric, subharmonic generation does not increase gradually with junction nonlinearity as harmonic generation does, but appears suddenly with a large amplitude when the microwave power level exceeds a certain threshold value depending on the junction losses whereas for $\omega \geq 2 \omega_{p}$ the amplitude grows up continuously from zero. The detailed calculations will not be reproduced here, but may be found in Ref. 3 .

From analog measurements where the waveform of the rf-voltage can be observed on an oscilloscope we know that the threshold value for subharmonic generation does not in general coincide with the $r . f$. voltage necessary for switching. Thus the theory of Ref. 3 is not directly applicable for a quantitative comparison, particularly with respect to the amplitude of the rf voltage and the power threshold curve. For this reason we have not tried to determine the magnitude and sign of the $\cos \phi_{\mathrm{o}}$-term ${ }^{12,13}$ although some 
information may be contained in the experimental curves.

A comment should be made to the appearance of the structure at $\omega / \omega_{p}=3 / 2$. This structure does not correspond to a higher-order solution of the Mathieu equation (3), but once the threshold curve corresponding to the excitation at $\omega \simeq 2 \omega_{\mathrm{p}}$ has been reached, it is known that $r$. f. voltage contains frequency components at $\omega / 2,3 \omega / 2,5 \omega / 2, \ldots$. . where the higher harmonics diminish rapidly in amplitude (Ref. 3 contains information about relative magnitudes for analog measurements). An interesting observation is that for a junction very similar to the one in Fig. 2 a but containing a short (circles in Fig. 4) the $3 / 2$ harmonics disappeared, whereas the other harmonics were essentially similar to the junction in Fig. 2a. This behavior may be expected if the $Q$ of the junction is lowered because of the short.

\section{EXPERIMENTAL DETAILS}

It is felt appropriate to show some details of the measuring setup. If the junction is biased $0.5 \%$ from the maximum supercurrent great care should be taken that the junction is switched by the applied rf voltage rather than by accidental transients. Transient voltage distribution and frequency will, of course, depend on the local mains supply, but the occurrance of numerous $1 \mathrm{kV}$ pulses per hour will not be atypical (switching a 60-W lamp may cause a $1-k V$ transient).

Since in general measuring setups are firmly grounded via extended metal systems, large transient currents will result in the loop thus formed if no precautions are taken (Fig. 5). The capacitors shown around the mains transformer are made up of winding capacitance, stray capacitance and now and then filter capacitors purposely applied by the equipment manufacturer.

The floating input/floating output amplifier shown in Fig. 5 reduces transient currents through the signal cable by a factor 10-100, depending on transient voltage rise-time. Furthermore, hum problems are largely eliminated.

Figure 6 shows schematically a metal body (heavy lines) containing bias current supply, input amplifier, stabilized power supply and (not shown) a temperature measuring circuit. Note, that no lead from the junction is brought outside the top flange. This ensures that junction leads and the above mentioned ground loop will have no impedance in common except for the impedance of the metal body itself.

The braid of the interconnecting cable (Fig. 5) is connected to the metal body (and chassis of the control section) through 18 pins equally spaced along the periphery of a 38-pole connector to maintain a certain degree of symmetry. This will to some extent reduce the mutual inductance between the signal path and the ground loop.

The microwaves were introduced into the cryostat through a low temperature coaxial cable and coupled to the junction by a loop surrounding the junction, with the junction placed in the middle of the loop.

\section{CONCLUSION}

By measuring switching curves for Jospe tunnel junctions, that is, the microwave power necesary to switch the junction to a finite voltage as a function of the dc-current in the zero voltage mode and analyze the position of the pronounced structure in the curves, it has been possible to identify the structure as belonging to subharmonic generation of the plasma frequency. This identification is further supported by similar measurements on a Josephson junction analog where the waveform of the $r . f$. voltage has been observed. Therefore, our conclusion is that we have demonstrated experimentally that the plasma resonance may be excited by parametric, subharmonic generation as predicted in Ref. 3. This motivates attempts at using a zero-voltage, i. e., dc-current biased, mode of a Josephson junction as the nonlinear element in a parametric amplifier.

At this point it should be emphasized, however, that the applicability may be limited by the power levels obtainable. In astronomical observations, where in general very weak signals containing no strong, coherent sources are investigated, a junction may operate quite satisfactory. In communication systems, however, the dynamic range may be a serious limitation. Let us consider a microwave receiver operating at $5 \mathrm{GHz}$ with the antenna looking into a $300-\mathrm{K}$ source. For a system bandwidth of only $10 \mathrm{MHz}$ thermal noise will be $-104 \mathrm{dbm}$, roughly equal to saturated output from the junction. This indicates, that a Josephson junction will be applicable in microwave systems only, if the operating frequency is very high bearing in mind the strong frequency dependence of saturated output power.

\section{ACKNOWLEDGMENT}

We wish to express our sincere thanks to Professor T. Stubb, Helsinki University of Technology, for placing the $\mathrm{Nb}$ junctions at our disposal.

\section{REFERENCES}

${ }^{1}$ P. Russer, Arch. Elek. Ubertragung 23, 417 (1969).

A. N. Vystavkin et al., Cornell Conference on High

Frequency Generation and Amplification 1971

3 (unpublished).

N. F. Pedersen, M. R. Samuelsen, and K. Saermark, J. Appl. Phys. 44, 5120 (1973).

${ }_{5}^{4} \mathrm{H}$. Zimmer, Appl. Phys. Letters 10, 193 (1967).

${ }^{5}$. Kanter and A. H. Silver, Appl. Phys. Letters 19, 515 (1971).

${ }^{6}$ R. Graeffe and T. Wiik, J. Appl. Phys. 42, 2146 (1971).

7 S. Shapiro, Phys. Rev. Letters 11, 80 (1963).

${ }^{8}$ A. J. Dahm, A. Denenstein, T. F. Finnegan,

D. N. Langenberg, and D. J. Scalapino, Phys. Rev.

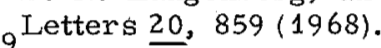

9 C. K. Bak and N. F. Pedersen, Appl. Phys. Letters $10 \frac{22}{2}, 149$ (1973).

O. P. Balkashin, L. J. Ostrovsky, and J. K. Yanson, Phys. of the Condensed State No. 28, 3 Khar'kov (1973).

${ }^{11}$ P. K. Hansma, G. I. Rochlin, and J. N. Sweet, Phys. Rev. B 노 3003 (1971). 
${ }^{12}$ N. F. Pedersen, T. F. Finnegan, D. N. Langenberg, Phys. Rev. B 5, 4151 (1972).

${ }^{13}$ C. M. Falco, $\bar{W}$. H. Parker, and S. E. Trullinger, Phys. Rev. Letters 31, 933 (1973); D. A. Vincent and B. S. Deaver, Jr., Phys. Rev. Letters 32, 212 (1974).

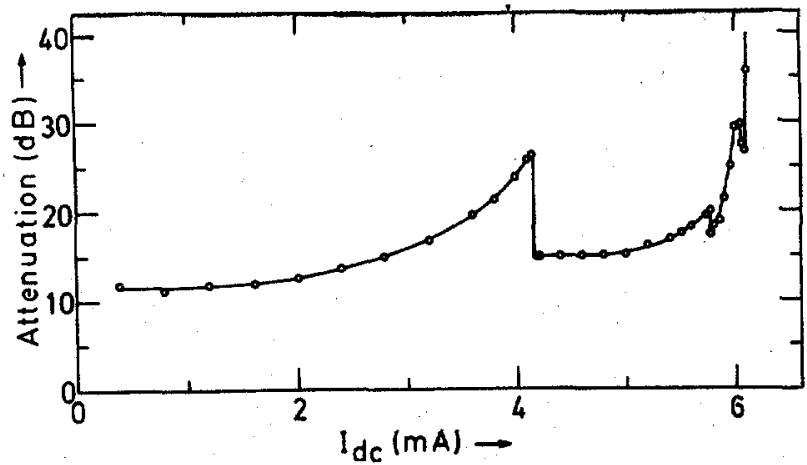

Fig. 1 Inserted attenuation in $\mathrm{db}$ of the applied microwave power necessary for switching the junction as a function of the dc-bias current $I_{d c}$ at zero voltage for a $\mathrm{Sn}-\mathrm{Sn}_{\mathrm{x}} \mathrm{O}_{\mathrm{y}}-\mathrm{Sn}$ tunnel junction.
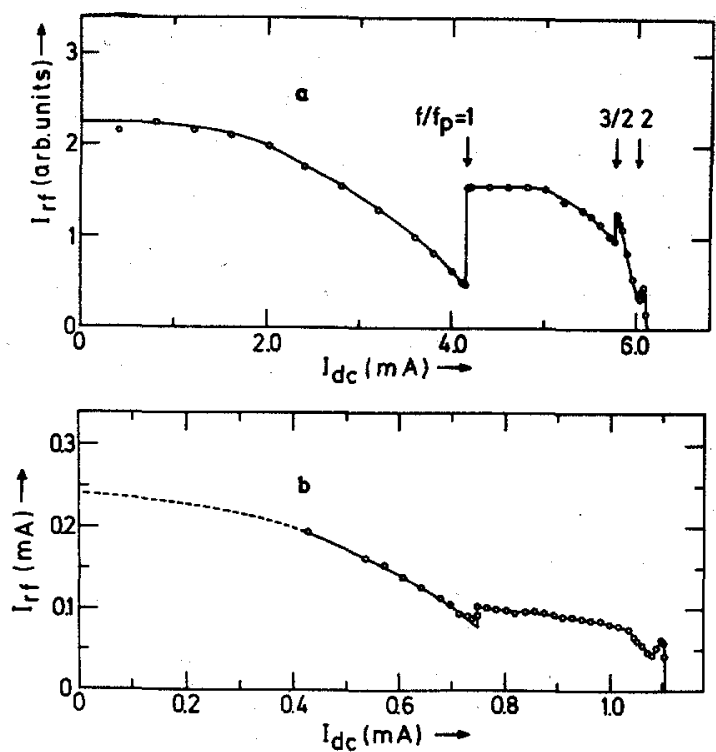

Fig. 2 The square root of the microwave power, i.e.,

a. the $r . f$. current in arbitrary units necessary for switching the junction as function of the dcbias current $I_{d c}$ at zero voltage for the same junction as in Fig. 1.

b. Similar curve as in a, but measured on a Josephson junction analog ( $I_{\mathrm{max}}=1.1 \mathrm{~mA}$, $R_{0}=500 \Omega, C=100 \mathrm{nF}, \mathrm{f}=11,2 \mathrm{kHz}$ ).

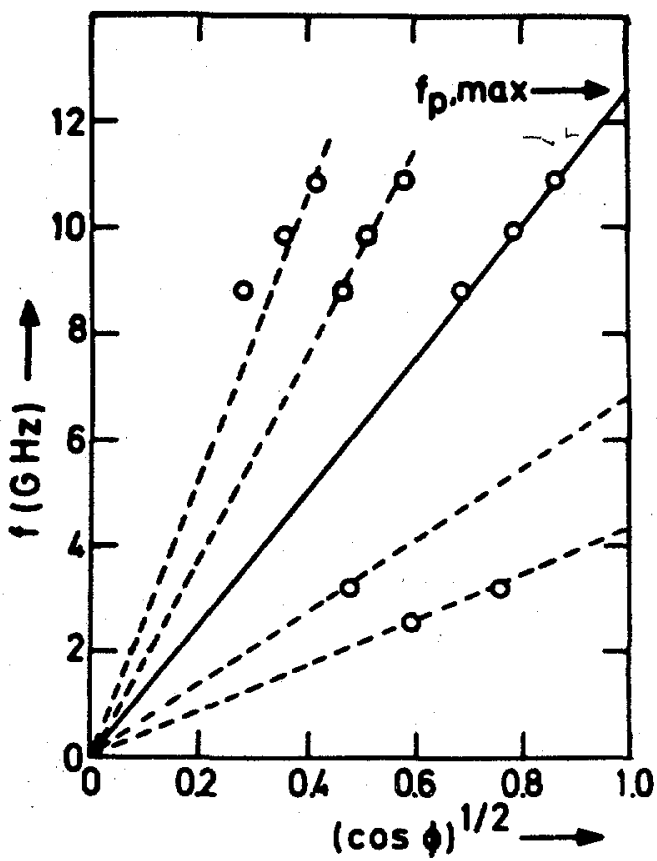

Fig. 3 The points in the diagram are the applied microwave frequency as a function of $\sqrt{\cos \phi}$ which is calculated from Eq. (2) using the dccurrent $\mathrm{I}_{\mathrm{d}}^{\mathrm{R}}$ corresponding to the pronounced structure in the switching curves. The extrapolated plasma frequency is $f_{\mathrm{p}, \max }=12.6 \mathrm{GHz}$. Note that $\sqrt{\cos \phi_{0}}=0.3$ corresponds to an $\mathrm{Idc}^{-}$ current of $99.5 \%$ of the maximum dc-current, $\mathrm{I}_{\mathrm{dc}}^{\max }$.

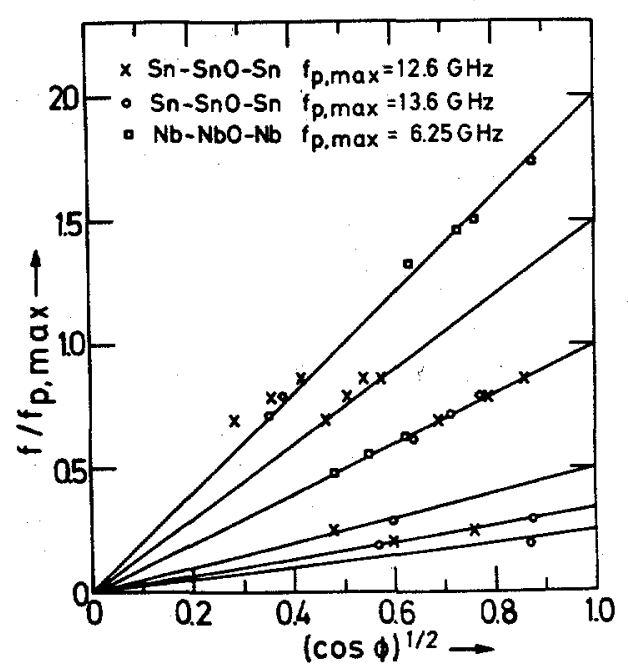

Fig. 4 Experimental points for three different tunnel junctions plotted in the same way as in Fig. 3, but the microwave frequency is here normalized to the plasma frequency found for each tunnel junction. 


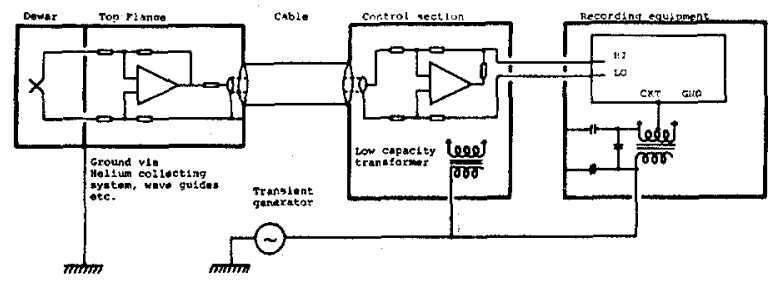

Fig. 5 Floating input/floating output amplifiers are used to break ground loops thus reducing transients from the mains supply to a safe leve1.

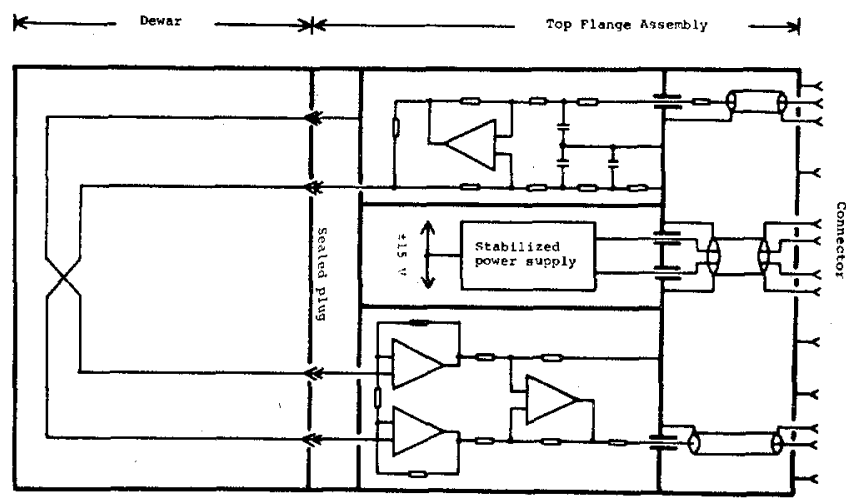

Fig. 6 Amplifier systems connected to the junction are housed in a metal enclosure directly on the top of the dewar. 